\title{
Functionalization of styrenic polymer through acylation and grafting under microwave energy
}

\author{
Marcos E Coustet and M Susana Cortizo
}

In this study, a new synthetic methodology for the functionalization of polystyrene (PS) has been developed, in which maleic anhydride was used in the presence of zinc oxide as catalyst and microwave energy was used as power source. A mechanism for the acylation reaction is proposed. The effect of reaction conditions on the degree of functionalization of PS was investigated. A subsequent reaction with diisopropyl fumarate/benzoyl peroxide was used to produce a graft copolymer. The polymers were identified and characterized by Fourier transform infrared spectroscopy, ultraviolet (UV) spectroscopy, proton nuclear magnetic resonance and size exclusion chromatography using double detection to confirm the structure of the macromolecules. In addition, the effect of acylation and grafting into PS on the corrosion resistance of steel surfaces were studied. The results show high efficiency of the acylation and grafting reactions under microwave conditions, with no PS degradation. Improvement in the corrosion resistance of both acylated and graft polymers suggest that they are suitable for use as protective coatings.

Polymer Journal (2011) 43, 265-271; doi:10.1038/pj.2010.131; published online 2 February 2011

Keywords: acylation; anhydride; graft copolymer; microwave synthesis; polystyrene; protective coating; zinc oxide

\section{INTRODUCTION}

Synthesis of new polymeric materials with multiple properties such as increased adhesion, tensile stress, high chemical inertia, color and good compatibility with additives or polar polymers is of great practical importance. ${ }^{1}$ With the exception of some cases, polymer blends generally show phase separation due to the incompatibility of the components. ${ }^{2}$ Thus, polymer synthesis with specific and relevant properties, such as those mentioned above, has been an important research goal in recent years. These materials can be prepared by copolymerization of different monomers or by chemical modification of appropriate polymers. However, the direct incorporation of the functional group by copolymerization of the functional comonomer has several drawbacks. For example, the Lewis acidity of heteroatoms can poison certain types of Ziegler-Natta or metallocene/ aluminoxano catalysts, and some functional groups can act as chain transfer agents ${ }^{3}$ that limit the obtained polymer molecular weight, resulting in deteriorating mechanical properties.

Thus, derivatization reactions have been proven to be the best way to obtain functionalized polymers. Especially, interesting are those that occur on the aromatic rings of polystyrene (PS) or its copolymers because these classes of macromolecules are susceptible to electrophilic aromatic substitution reactions. ${ }^{4-6}$ Among these reactions, FriedelCraft acylation is an important type of reaction because it enables the introduction of carbonyl or carboxyl groups depending on the reagent used. Several polymers containing aromatic rings, such as PS, have been acylated using Lewis acids catalysts, showing improvements in thermal stability, impact stress and corrosion resistance. ${ }^{7}$ Syndiotactic PS functionalized with succinic anhydride and $\mathrm{Cl}_{3} \mathrm{Al}$ showed lower melting and crystallization temperatures and a lower degree of crystallization. ${ }^{8}$ A commercial poly(styrene-b-ethylene-co-propylene) was modified into an ionomer by carboxylation using acetyl chloride/ $\mathrm{Cl}_{3} \mathrm{Al}$ and subsequent oxidation with sodium hypochlorite. ${ }^{9}$ Lewis acid catalysis was used in the aforementioned work, but this technique has serious drawbacks, such as crosslinking reactions, polymer degradation and frequently colored products that are indicative of unwanted side reactions. ${ }^{10,11}$ It is therefore necessary to seek out new methods using mild acylation conditions.

An additional problem associated with the use of Lewis catalysts is that such catalysts are not easily recovered and recycled, thereby generating large amounts of toxic waste. In recent years, Friedel-Craft reactions using solid catalysts, such as zeolites, sulfated zirconia and metal oxides, have proven to be effective. ${ }^{12-14}$ Some of these reactions on different organic compounds have been tested under microwave heating conditions, showing improved performance compared with reactions carried out under thermal heating conditions. The reactions carried out using microwave energy have many advantages: increased yield, reduced reaction times, greater selectivity with respect to obtained product (in some cases) and more energy savings, among others. This methodology demonstrated improvements (higher yield, shorter reaction times) in various reactions of polymer chemistry. ${ }^{15-18}$

The present work was undertaken to study the acylation of PS with maleic anhydride (MA) in the presence of zinc oxide under microwave

Laboratorio de Estudio de Compuestos Orgánicos (LADECOR), Instituto de Investigaciones Fisicoquímicas Teóricas y Aplicadas (INIFTA), Facultad de Ciencias Exactas, Universidad Nacional de La Plata, La Plata, Argentina

Correspondence: Dr MS Cortizo, Quimica, Laboratorio de Estudio de Compuestos Orgánicos (LADECOR), Instituto de Investigaciones Fisicoquímicas Teóricas y Aplicadas (INIFTA), Facultad de Ciencias Exactas, Universidad Nacional de La Plata, Dg 113 y 64, CCT-La Plata. CC 16 Sucursal 4, Buenos Aires 1900 , Argentina.

E-mail: gcortizo@inifta.unlp.edu.ar

Received 2 August 2010; revised 13 November 2010; accepted 14 November 2010; published online 2 February 2011 
conditions. The microwave energy was evaluated as the product of the power and time of irradiation. In particular, the aim is to investigate the effect of irradiation power and the ratio of reactants on the structure and properties of the resulting functionalized polymer. In addition, a graft copolymer was obtained by reaction of acylated PS with diisopropyl fumarate (DIPF). The application of graft copolymer as a protective coating against corrosion was also investigated.

\section{EXPERIMENTAL PROCEDURE}

\section{Materials}

MA was donated by Maleic SA (Argentina), benzoyl peroxide was recrystallized from methanol and zinc oxide $(\mathrm{ZnO})$, which was purchased from Aldrich (Buenos Aires, Argentina). Other solvents were purchased from Merck and Sintorgan (PA) (Buenos Aires, Argentina).

Three samples of PS polymers of different weight-average molecular weights $\left(M_{\mathrm{w}}\right)$ and molecular weight distributions were synthesized by radical polymerization initiated by benzoyl peroxide under thermal or microwave conditions. ${ }^{17}$ The molecular weight distribution was evaluated as the ratio of $M_{\mathrm{w}} / M_{\mathrm{n}}$, where $M_{\mathrm{n}}$ is the number average molecular weight. The polymers were designated as PS1 $\left(M_{\mathrm{w}}=66500 \mathrm{~g} \mathrm{~mol}^{-1}, M_{\mathrm{w}} / M_{\mathrm{n}}=2.2\right)$, PS2 $\left(M_{\mathrm{w}}=38800 \mathrm{~g} \mathrm{~mol}^{-1}, M_{\mathrm{w}} /\right.$ $\left.M_{\mathrm{n}}=2.9\right)$ and PS3 $\left(M_{\mathrm{w}}=25900 \mathrm{~g} \mathrm{~mol}^{-1}, M_{\mathrm{w}} / M_{\mathrm{n}}=2.2\right)$. The polymers were isolated and purified by a common procedure of dissolution/precipitation using a toluene/methanol mixture in the ratio of 1:5.

\section{Acylation under microwave conditions}

A conical 50-ml Pyrex flask reactor was used to carry out the reaction. MA and $\mathrm{ZnO}$ were added to $2 \mathrm{ml}$ of PS solution in nitrobenzene $\left(0.1 \mathrm{~g} \mathrm{ml}^{-1}, 1 \mathrm{mmol}\right.$ based on benzene ring per $\mathrm{ml}$ ). The weight relationship of $\mathrm{MA}$ and $\mathrm{ZnO}$ was varied with respect to PS. The reactor was stopped with a septum and introduced into a domestic microwave oven (White-Westing House, La Plata, Buenos Aires, Argentina), which has a maximum power of $700 \mathrm{~W}$. Three different power settings and heating time combinations were tested: 350,560 and $700 \mathrm{~W}$ at 4 , 6 and $8 \mathrm{~min}$, respectively. The sample temperature was measured by a mercury thermometer at the end of the reaction and recorded in $<10 \mathrm{~s}$, following the technique previously published. ${ }^{17}$ The temperature data obtained at each power irradiation are explained in the Results and Discussion section. The product was isolated by adding $5 \mathrm{ml}$ of methanol followed by centrifugation. The polymer was purified by toluene dissolution, filtration (separation of $\mathrm{ZnO}$ ) and methanol precipitation (toluene to methanol, 1:5). Preliminary tests conducted to select the polymerization conditions showed that the reactions were reproducible. It should be noted that the catalyst can be recovered by simply washing this with $\mathrm{Cl}_{2} \mathrm{CH}_{2}$, resulting in a more cost-effective process. ${ }^{19}$

To compare the previous methodology to the current work, a thermal reaction was carried out at $139^{\circ} \mathrm{C}$ for $60 \mathrm{~min}$ using a thermal bath and the following concentration ratios: $[\mathrm{ZnO}] /[\mathrm{MA}]=0.5$ and $[\mathrm{PST}] /[\mathrm{MA}]=1$. The choice of temperature was based on the previously measured temperature in the test performed under microwave conditions ( 4 min at $560 \mathrm{~W}$ ).

\section{Graft reaction on the functionalized PS}

In an Erlenmeyer flask $(25 \mathrm{ml})$ containing $0.1 \mathrm{~g}$ of functionalized polystyrene (PSF1), $0.5 \mathrm{ml}(0.494 \mathrm{~g})$ of DIPF and $7.26 \mathrm{mg}(60 \mathrm{~mm})$ of benzoyl peroxide as radical initiator were added. The flask was stopped with a septum and purged with $\mathrm{N}_{2}$ for $30 \mathrm{~min}$. Then the flask with the sample was placed in the center of the turntable of the microwave oven and irradiated at $140 \mathrm{~W}$ for $15 \mathrm{~min}$. These conditions were selected based on the previous study of homopolymerization of DIPF. ${ }^{17}$ After the treatment, the mixture was allowed to cool to room temperature, followed by the isolation and purification of the polymer by solubilization-precipitation (toluene/methanol, 1:5). The conversion of monomer $(\mathrm{C} \%)$ and grafting degree (GD \%) was determined gravimetrically and calculated using the following equations:

$$
\begin{aligned}
& \mathrm{C} \%=\left(W_{2}-W_{1}\right) 100 / W_{o} \\
& \mathrm{GD} \%=\left(W_{2}-W_{1}\right) 100 / W_{1}
\end{aligned}
$$

where $W_{\mathrm{o}}, W_{1}$ and $W_{2}$ are the mass of the monomer, the mass of the initial PSF and the mass of the obtained graft copolymer, respectively.

The same reaction was also performed using conventional thermal heating method. In this case, the reaction was carried out in a degassed glass tube maintained at $85^{\circ} \mathrm{C}$ for $20 \mathrm{~h}$. The isolation and purification of the product was the same as previously described. The choice of temperature was based on the previously measured temperature in the test performed under microwave conditions $(15 \mathrm{~min}$ at $140 \mathrm{~W})$.

\section{Characterization techniques}

Fourier transform infrared spectra of the polymer films deposited onto a sodium chloride $(\mathrm{NaCl})$ window were recorded on a Nicolet 380 FTIR (Thermo Electron Corporation, Madison WI, USA) between $4000-400 \mathrm{~cm}^{-1}$ with a resolution of $4 \mathrm{~cm}^{-1}$ and 32 accumulated scans. The EZ-OMNIC software (EZOMNIC 7.4.127, Thermo Fisher Scientific Inc, Madison, WI, USA) was used to analyze the spectra. The films were prepared by the solventcasting method: first, a polymer solution in chloroform $(5 \%(\mathrm{w} / \mathrm{v}))$ was prepared and poured onto the $\mathrm{NaCl}$ window. Then the solvent was allowed to evaporate at room temperature, with the resulting films dried under vacuum until the weight remained constant.

Absorption spectrum was obtained using an Agilent 8453E UV-visible spectroscopy (Agilent Technologies, Santa Clara, CA, USA) system at room temperature, using chloroform as the solvent $\left(c=10 \mathrm{mg} \mathrm{ml}^{-1}\right)$.

Proton nuclear magnetic resonance $\left({ }^{1} \mathrm{H}-\mathrm{NMR}\right)$ spectra of polymers were recorded on a Variam-200 MHz (Mercury 200; Agilent Technologies) at $35^{\circ} \mathrm{C}$ in $\mathrm{CDCl}_{3}$. Tetramethylsilane (TMS) was used as an internal standard.

The average molecular weight and the molecular weight distribution were determined by size exclusion chromatography (SEC) in a LKB-2249 instrument (Pharmacia LKB, Uppsala, Sweden) at $25^{\circ} \mathrm{C}$. A series of $\mu$-Styragel columns (Millipore (Waters Chrom. Div.), Milford, MA, USA), with pore sizes of $10^{5}$, $10^{4}, 10^{3}$, and $100 \AA$ were used with chloroform as an eluent. The polymer concentration was $4-5 \mathrm{mg} \mathrm{ml}^{-1}$, and the flow rate was $0.5 \mathrm{ml} \mathrm{min}^{-1}$. The polymer was analyzed using double detection as previously reported. ${ }^{20}$ Mass chromatograms of the polymers were detected by a Shimadzu (SPD-10A) UV/ VIS detector (Shimadzu Corporation, Tokyo, Japan) at $254 \mathrm{~nm}$ (for the phenyl group), whereas the carbonyl group from the derivatization of the phenyl rings was detected by infrared (IR) absorption at $5.75 \mu \mathrm{m}$ with a Miram IA spectrophotometer detector (Foxboro Analytical, South Nor Walk, CT, USA). The calibration was done with PS standards supplied by Polymer Laboratories (Polystyrene Kit, Alltech Associates, Inc, Deerfield, IL, USA) and Polysciences, Inc (Warrington, PA, USA).

\section{Determination of corrosion resistance}

Thin polymer films were formed from polymer solutions $(10 \mathrm{w} / \mathrm{v} \%$ in chloroform) over steel pieces with dimensions of $10 \times 20 \times 1 \mathrm{~mm}^{3}$. They were then exposed to $\mathrm{NaCl}$ solution $(3 \mathrm{w} / \mathrm{v} \%)$ in open air for 10 days, and subsequently removed from these environments ( $\mathrm{NaCl}$ solution). Surfaces were monitored using an optical microscope (BX51 model-Olympus (Olympus Corp., Tokyo, Japan) and DP Controller image processor (DP71 Olympus Corp.) before and after putting them in the saline solution.

\section{RESULTS AND DISCUSSION}

\section{Synthesis of PSF}

The product of the reaction between PS and MA catalyzed by $\mathrm{ZnO}$ under microwave condition is a copolymer, as shown in Scheme 1.

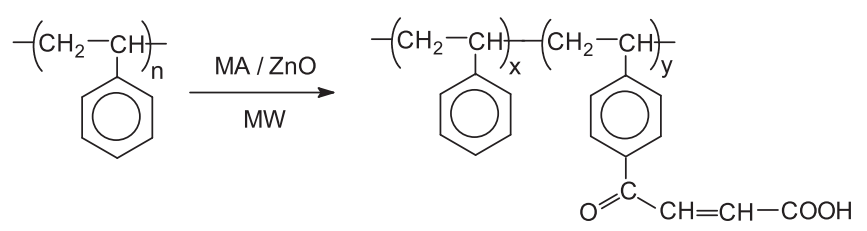

Scheme 1 Synthesis of functionalized polystyrene (PSF) starting with polystyrene (PS), maleic anhydride (MA) and zinc oxide ( $\mathrm{ZnO})$ as catalysts under microwave (MW) conditions. 


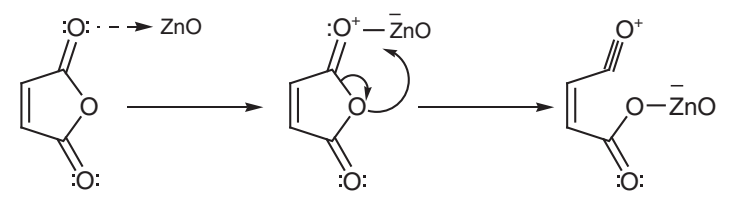

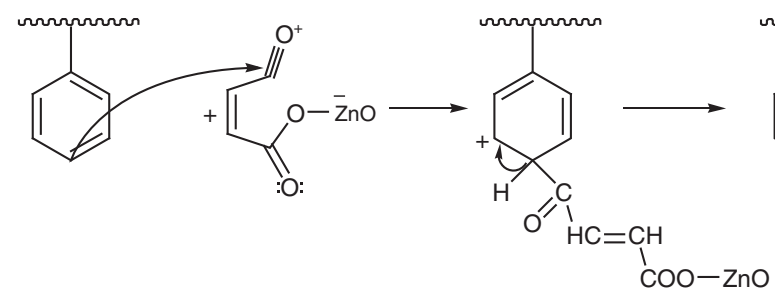<smiles>COc1ccc(C(=O)C=CC(=O)O[Na])cc1</smiles><smiles>CCOc1ccc(C(=O)/C=C/C(=O)O)cc1</smiles>

Scheme 2 Proposed mechanism for the acylation of polystyrene with maleic anhydride and zinc oxide $(\mathrm{ZnO})$ as catalysts.

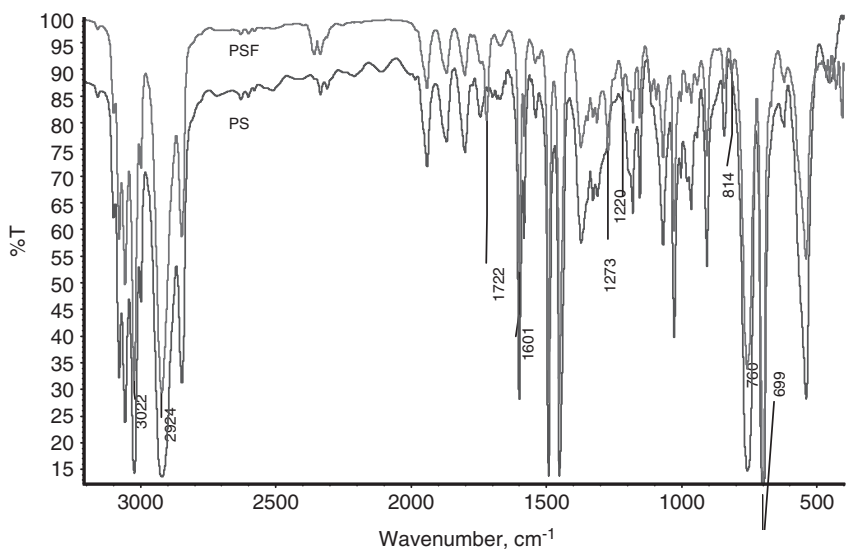

Figure 1 Fourier transform infrared spectra of the polystyrene (PS) sample and the functionalized polystyrene (PSF) sample.

The proposed mechanism of the reaction, based on the knowledge of the Friedel-Craft reactions, is proposed as the following (Scheme 2). The first stage consists of the coordination of $\mathrm{Zn}$ to the free electron of the oxygen on the anhydride, which induces the formation of the acylium ion, a reactive species. Then the addition of acylium ion to the phenyl ring of PS breaks off the hydrogen atom and finally recovers the aromaticity of the phenyl ring.

The characterization and identification of PSF was carried out by different spectroscopic methods to fully confirm their structure. Figure 1 shows the Fourier transform infrared spectra of the acylated and original PS. It is possible to see the apparition of new characteristic bands at 1722.5, 1670 (corresponding to $\mathrm{vC}=\mathrm{O}$ ), 1272.9 (corresponding to $\delta \mathrm{O}-\mathrm{H}$ ), 1108.3 (corresponding to $\mathrm{vC}-\mathrm{O}$ ), 690 (corresponding to $\delta \mathrm{C}-\mathrm{H}$ and $\mathrm{CH}=\mathrm{CH}$ cis) and 814.5 (corresponding to $\delta \mathrm{C}-\mathrm{H}$ Ar p-substituted) $\mathrm{cm}^{-1}$, which show that the acylation reaction of the aromatic rings is effective. Similar evidence was found by other researches using different synthetic conditions. ${ }^{7,8}$

Ultraviolet (UV) absorption measurements were made because it is known that a substituted group affects the electronic and optical properties of a polymer by its electronic nature and size. ${ }^{21}$ Figure 2 shows the absorption spectra of PS and PSF, which demonstrates the hyperchromic effect on the $\mathrm{B}$ band characteristic of the benzene ring, attributed to the $\mathrm{CO}-\mathrm{CH}=\mathrm{CH}-\mathrm{COOH}$ group introduced by structural modifications.

The unequivocal structural identification was obtained by ${ }^{1} \mathrm{H}-\mathrm{NMR}$ analysis. Figure $3 \mathrm{a}$ shows the ${ }^{1} \mathrm{H}-\mathrm{NMR}$ spectra of PSF1 and the corresponding assignments. The signals at 1.49 and

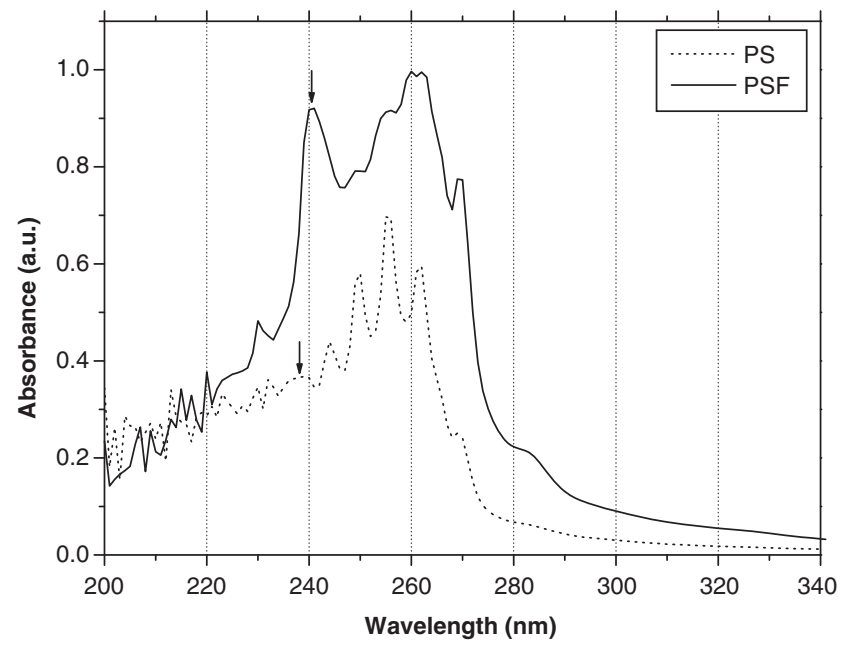

Figure 2 UV absorption spectra of polystyrene (PS, shown as dotted line) and functionalized polystyrene (PSF, shown as solid line) in $10 \mathrm{mg} \mathrm{ml}^{-1}$ chloroform solutions.

1.90 p.p.m. correspond to methylene and methyne groups of the main chain, whereas the signals at 6.6 and 7.1 p.p.m. include both the resonance of vinyl methyne and aromatic hydrogen. The signal at 7.8 p.p.m. was attributed to an $\mathrm{H}$ bond in the ortho position to the $\mathrm{CO}-\mathrm{CH}=\mathrm{CH}-\mathrm{COOH}$ group, indicating that the substitution occurred exclusively at the para position of the aromatic ring. Similar results were found by other investigators. ${ }^{22,23}$

The SEC analysis of this product was carried out using double detection. The UV-visible detector response (at $254 \mathrm{~nm}$ ) indicated the absorption of the aromatic group and was proportional to polymer concentration. The IR detector, set to $5.75 \mu \mathrm{m}\left(1720 \mathrm{~cm}^{-1}\right.$ in wave numbers), detected the carbonyl group absorption. Figure 4a shows a representative chromatogram where significant signals can be observed by both detectors. The presence of two signals in the chromatogram of the PSF is additional evidence that the acylation reaction has occurred. The signal of carbonyl group appear slightly shifted to higher elution volume (representing smaller molecular weight), suggesting that the acylation is favored in smaller molecular size fractions, as will be shown below.

\section{Effect of irradiation power}

To determine the optimal conditions for the acylation reaction of PS, the effect of different experimental variables on the derivatization degree was studied. The degree of functionalization (DF) of PS can be 

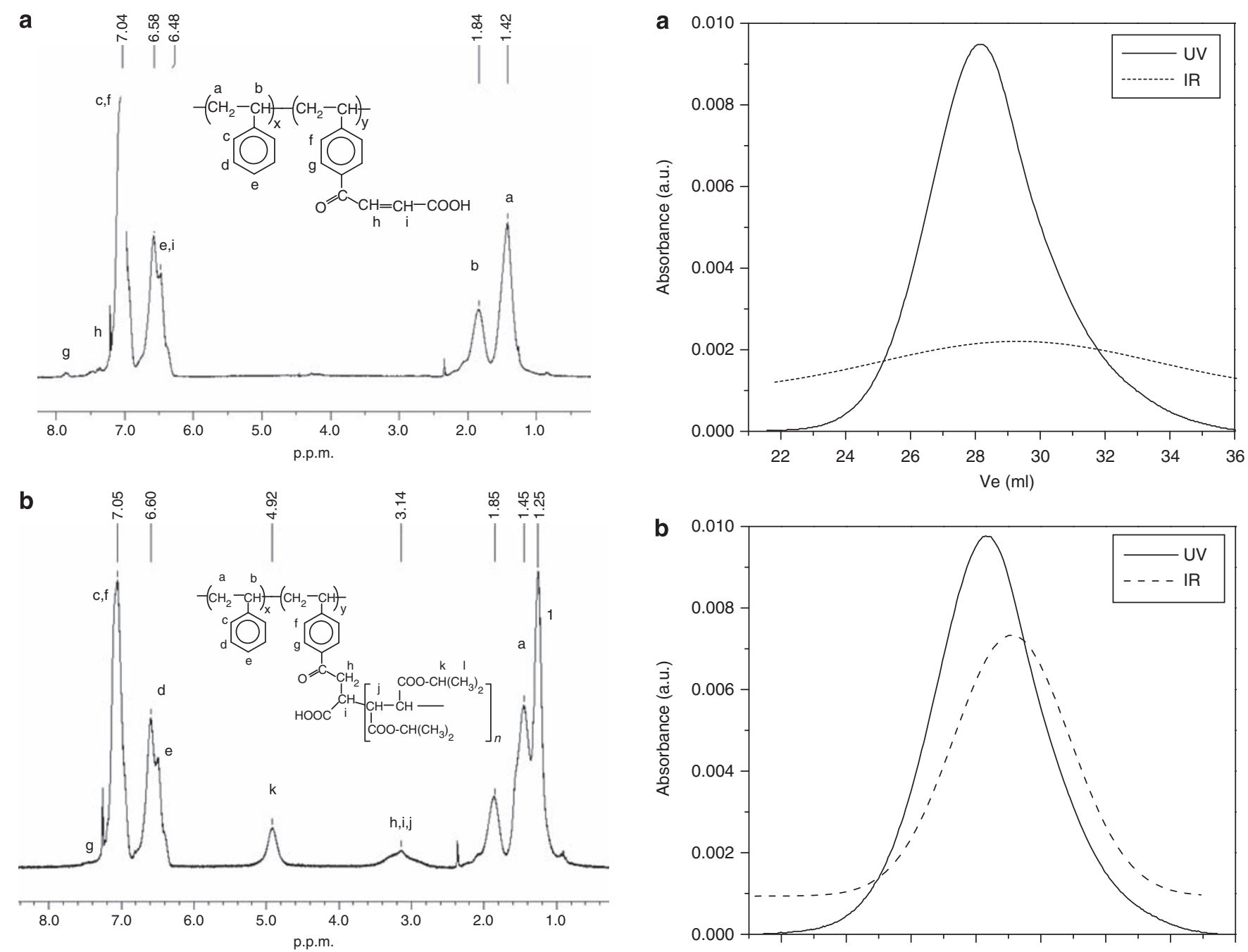

Figure $3{ }^{1} \mathrm{H}-\mathrm{NMR}$ spectra $\left(\mathrm{DCl}_{3} \mathrm{C}\right)$ for (a) functionalized polystyrene (PSF1) and (b) grafted polymer (PSF1g).

estimated from the relative intensities of the respective signals in ${ }^{1} \mathrm{H}-\mathrm{NMR}$ spectrum according to the following equation:

$$
\mathrm{DF}(\%)=3 I(\mathrm{~g}) \times 100 / 2[I(\mathrm{a})+I(\mathrm{~b})]
$$

where $I(\mathrm{a}), I(\mathrm{~b})$ and $I(\mathrm{~g})$ are the resonance integrals of the peak shown in Figure 3a.

Figure 5 shows the effect of irradiation power on the DF. The relationship between the polymer and the anhydride (1:1) and the relationship between the catalyst and the acylant (1:2) remained constant. Figure 5 also shows the changes in the temperature measurement at each irradiation power setting: 136,139 and $148^{\circ} \mathrm{C}$ corresponding to 350,560 and $700 \mathrm{~W}$, respectively. The increase of the reaction temperature was proportional to the irradiation power. Therefore, the graph shows an increase in the DF with an increase in irradiation power and temperature.

Although a slightly lower $\mathrm{DF}$ ( $\mathrm{DF}=4.5 \pm 0.1$ versus $6.2 \pm 0.2$, see run 2 in Table 1) was observed for the reaction carried out under thermal conditions $\left(139^{\circ} \mathrm{C}, 60 \mathrm{~min}\right)$, it is noteworthy that the reaction times decreased when the reaction was carried out under microwave conditions (60 versus $4 \mathrm{~min}$ under thermal and microwave conditions, respectively). The DF for functionalized polystyrene synthesized under thermal conditions was evaluated from the relative intensities of the

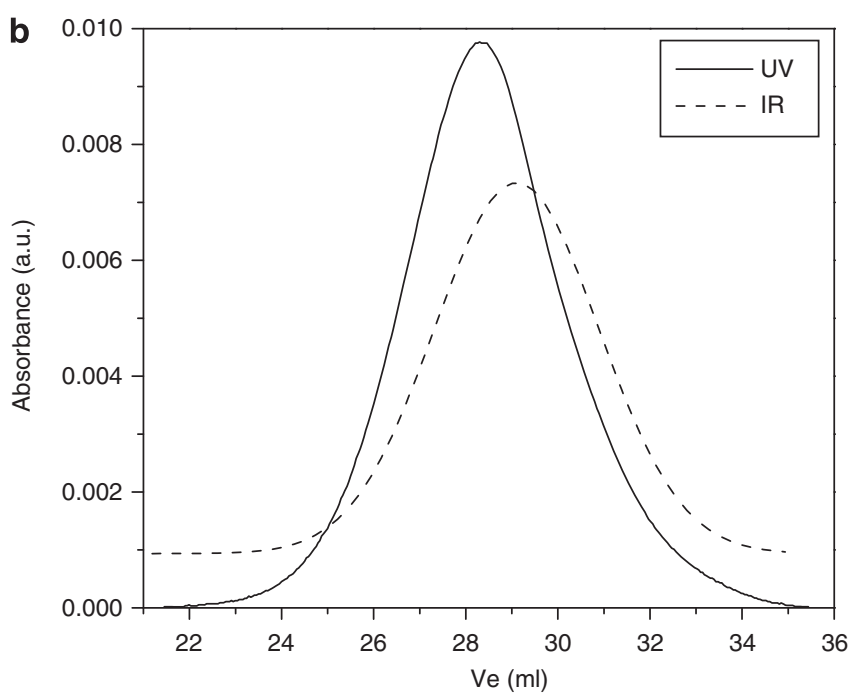

Figure 4 Chromatographic profiles for (a) functionalized polystyrene (PSF1), with reaction conditions of $[\mathrm{PS} 1] /[\mathrm{MA}]=1,[\mathrm{ZnO}] /[\mathrm{MA}]=0.5,560 \mathrm{~W}, 4 \mathrm{~min}$; (b) grafted polymer (PSF1g) starting with PSF1. The UV-visible detector response is shown as solid line and the infrared detector response is shown as dotted line in both elugrams.

respective signals in ${ }^{1} \mathrm{H}-\mathrm{NMR}$ spectrum (see Supplementary information), as above. This result demonstrates the greater efficiency of the process using microwave energy. The average molecular weight and molecular weight distribution of functionalized polymer obtained under thermal conditions do not shown significant differences with the initial values $\left(M_{\mathrm{w}}=66000 \mathrm{~g} \mathrm{~mol}^{-1}, M_{\mathrm{w}} / M_{\mathrm{n}}=2.2\right)$. The signal of the IR detector was very low due to a lower DF (not shown).

\section{Effect of the reagents ratio}

The dependence of DF on the $[\mathrm{ZnO}] /[\mathrm{MA}]$ ratio was investigated during the reaction carried out at $560 \mathrm{~W}$ for $4 \mathrm{~min}$. Table 1 shows the optimal conditions for the acylation reaction for which the ratio between the $\mathrm{ZnO}$ and MA concentrations was 0.5 (run 2). Similar results were found during the acylation of alcohols and amines with this catalyst. ${ }^{19}$

However, DF shows a small increase with increasing [PS]/[MA] ratio (run 2, 4, 5 and 6). This behavior is likely related to the availability of the aromatic rings of PS and its interaction with the 


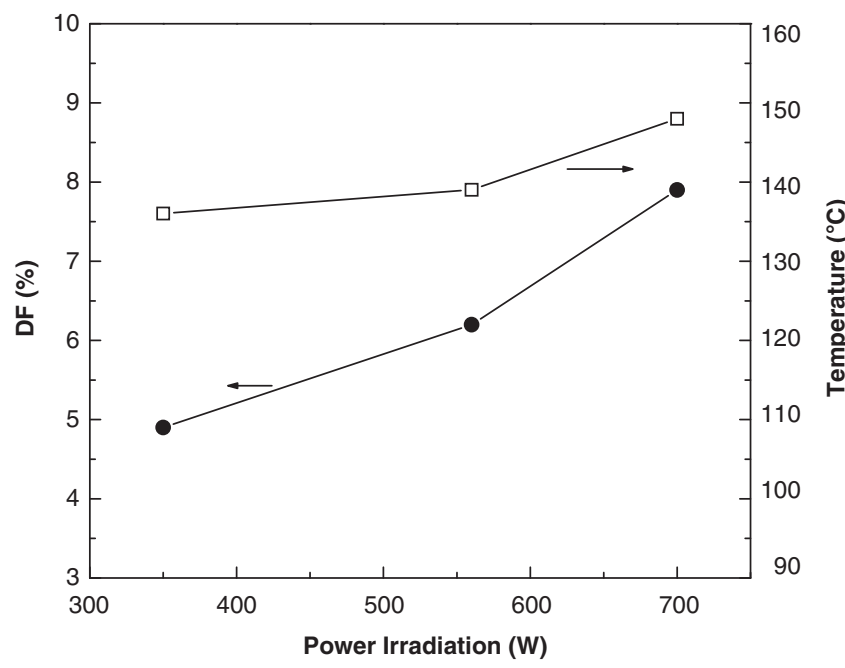

Figure 5 Effect of the irradiation power on the degree of functionalization (DF, \%) of polystyrene (PS1, shown as circles) and the relationship between irradiation power and reaction temperature (shown as squares). Reaction conditions: $[\mathrm{PS} 1] /[\mathrm{MA}]=1,[\mathrm{ZnO}] /[\mathrm{MA}]=0.5,4 \mathrm{~min}$.

Table 1 Effect of the [ZnO]/[MA] and [PS]/[MA] ratio on the degree of functionalization (DF) of one sample of polystyrene (PS1)

\begin{tabular}{lccc}
\hline Run & {$[\mathrm{ZnO}] /[\mathrm{MA}]$} & {$[\mathrm{PS}] /[\mathrm{MA}]$} & DF $(\%) \pm$ s.e.m. \\
\hline 1 & 0.25 & 1.0 & $3.4 \pm 0.1$ \\
2 & 0.5 & 1.0 & $6.2 \pm 0.2$ \\
3 & 1.0 & 1.0 & $3.5 \pm 0.1$ \\
4 & 0.5 & 0.5 & $5.7 \pm 0.2$ \\
5 & 0.5 & 2.0 & $7.5 \pm 0.1$ \\
6 & 0.5 & 3.0 & $7.8 \pm 0.1$ \\
\hline
\end{tabular}

Reaction conditions: 560 W, 4 min.

${ }^{\mathrm{a}} n=5$.

Table 2 Effect of the initial weight-average molecular weight $\left(M_{\mathrm{w}}{ }^{i}\right)$ of polystyrene on the degree of functionalization (DF) and the macromolecular characteristics of the final polymer

\begin{tabular}{lccccc}
\hline Sample & $\mathrm{M}_{w}{ }^{i}\left(\mathrm{gmol}^{-1}\right)$ & $\mathrm{M}_{w} \mathrm{M}_{n}{ }^{i}$ & DF (\%) \pm s.e.m. & $\mathrm{M}_{w}{ }^{f}\left(\mathrm{gmol}^{-1}\right)$ & $\mathrm{M}_{w} \mathrm{M}_{n}{ }^{f}$ \\
\hline PS1 & 66500 & 2.2 & $6.2 \pm 0.2$ & 62300 & 2.2 \\
PS2 & 38800 & 2.9 & $7.7 \pm 0.1$ & 27800 & 2.4 \\
PS3 & 25900 & 2.2 & $8.5 \pm 0.1$ & 17500 & 1.9 \\
\hline
\end{tabular}

$M_{\mathrm{w}}{ }^{\dagger}$ and $M_{\mathrm{w}} / M_{\mathrm{n}}{ }^{\dagger}$ are the weight-average molecular weight and the polydispersity index of the $M_{\mathrm{w}}{ }_{\mathrm{w}}$ and $M_{\mathrm{w}} / M_{\mathrm{n}}$ are the weight-average molecular weight and the polydispersity index of the
acylated polystyrene (PS), respectively. Reaction conditions: [PS]/[MA] $=1,[\mathrm{ZnO}] /[\mathrm{MA}]=0.5$, $560 \mathrm{~W}, 4 \mathrm{~min}$.

electrophile, as a result of the conformation of the macromolecule in solution.

It should be noted that other researchers who studied acylation reactions used acid chlorides as the acylant and $\mathrm{ZnO}$ as the catalyst. Their results indicated that the reaction was not possible with acid anhydrides, which is contrary to what is shown in this work. ${ }^{19}$

Effect of the weight-average molecular weight of PS

The interest in analyzing the effect of $M_{\mathrm{w}}$ on the efficiency of the derivatization reaction is due to its technological importance. $M_{\mathrm{w}}$ is known to significantly influence the physical and mechanical properties of the final product. Three PS samples with different $M_{\mathrm{w}}$ and distributions were compared. Table 2 shows the results of the acylation

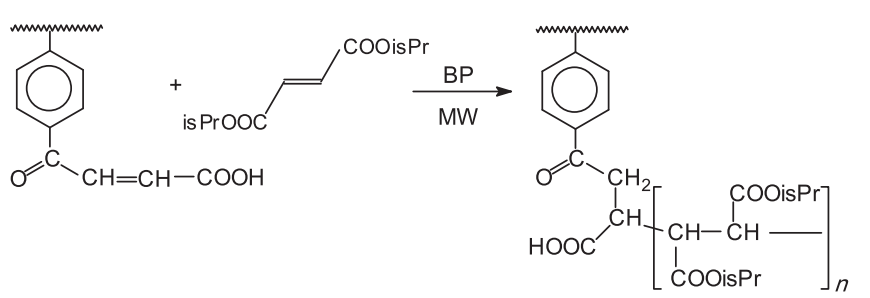

Scheme 3 Grafting reaction on functionalized polystyrene with diisopropyl fumarate monomer and benzoyl peroxide (BP) as the initiators under microwave energy (MW).

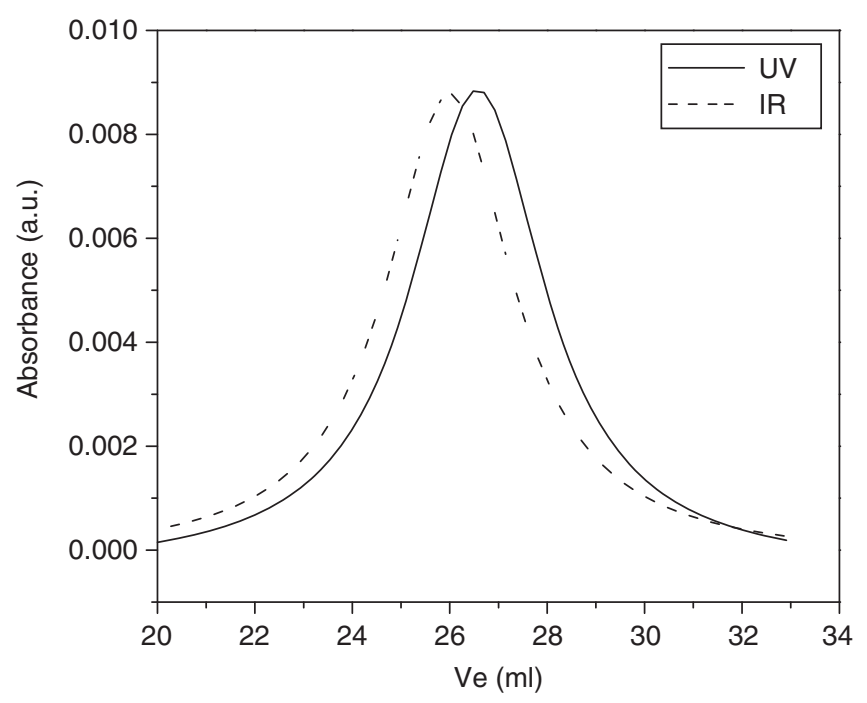

Figure 6 Chromatographic profiles for grafted polymer (PSF1g) starting with PSF1. Reaction conditions: $[\mathrm{PS} 1] /[\mathrm{MA}]=1,[\mathrm{ZnO}] /[\mathrm{MA}]=0.5,85^{\circ} \mathrm{C}, 20 \mathrm{~h}$. The UV-visible detector response is shown as a solid line, and the infrared detector response is shown as a dotted line.

reaction of PS1 under the following conditions: $[\mathrm{PS}] /[\mathrm{MA}]=1$, $[\mathrm{ZnO}] /[\mathrm{MA}]=0.5$, with $4 \mathrm{~min}$ of $560 \mathrm{~W}$ microwave irradiation. Here, $M_{\mathrm{w}}{ }^{\mathrm{i}}$ and $M_{\mathrm{w}}{ }^{\mathrm{f}}$ are the weight-average molecular weight of PS before and after the derivatization reaction, respectively. As shown in the table, when the $M_{\mathrm{w}}$ decreased, the functionalization degree increased, indicating that more functional groups were associated with the lower molecular size. On the other hand, the $M_{\mathrm{w}}$ of the acylated PS decreased somewhat with the degree of derivatization. This behavior may be attributed to a more compact conformation of PSF than the parent PS in the hydrophobic solvent $\left(\mathrm{Cl}_{3} \mathrm{CH}\right)$ due to the formation of hydrophilic domains. Further, the degradation of the polymer was not significant during the reaction because the polydispersity index did not increase. A similar result was observed for the acylation of ethylene and allylbenzene copolymers with different anhydrides. ${ }^{22}$

Graft reaction on the functionalized polystyrene

The presence of a $\mathrm{C}=\mathrm{C}$ bond on the acylated $\mathrm{PS}$ allows a radical grafting reaction. This is a very versatile reaction that introduces a pendent chain of varying nature. Such reactions were carried out starting with DIPF. This monomer was selected because its radical homopolymerization under microwave irradiation was systematically studied in our laboratory. ${ }^{17}$ The reaction produces a graft copolymer consisting of a poly(isopropoxycarbonylmethylenic) pendent chain as shown in Scheme 3. The graft copolymer (PSF1g) was identified by ${ }^{1} \mathrm{H}-\mathrm{NMR}$, as shown in Figure $3 \mathrm{~b}$, with the main characteristic signals at the following positions $\left(\delta_{\mathrm{H}}\right.$, p.p.m.): $4.92(-\mathrm{OC}-\mathrm{H}<$, isopropyl 
a

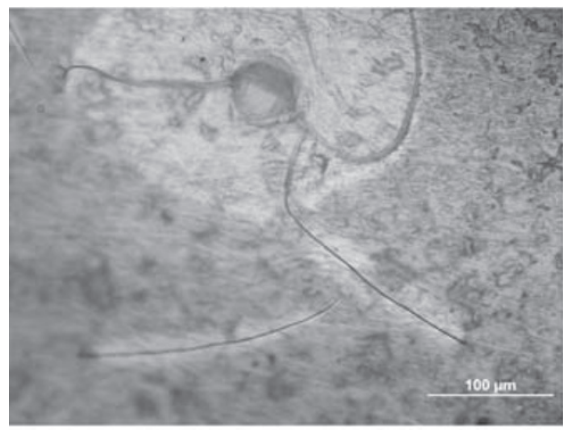

C

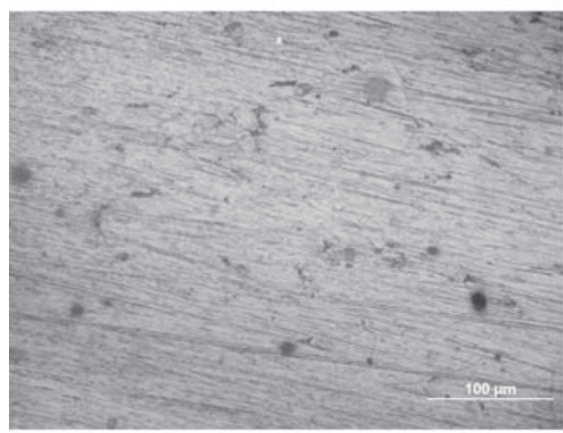

e

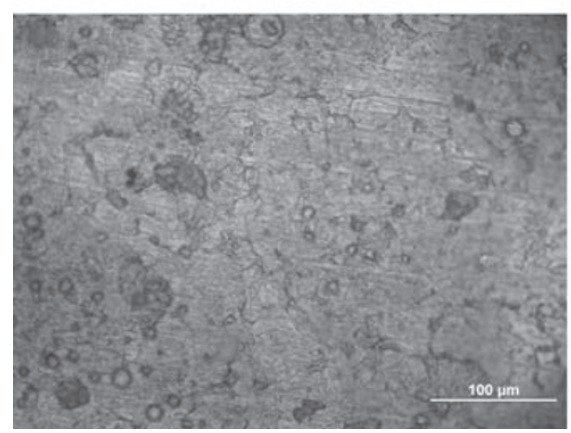

b

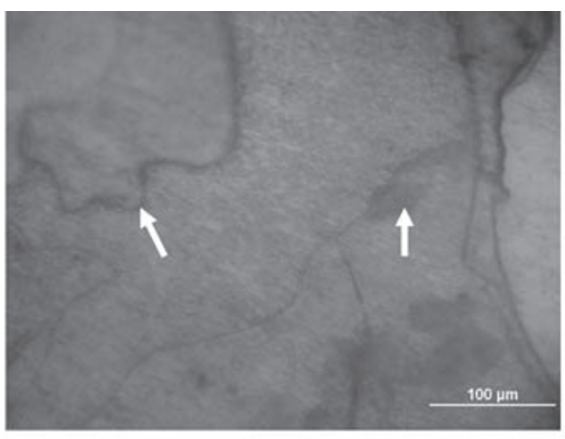

d

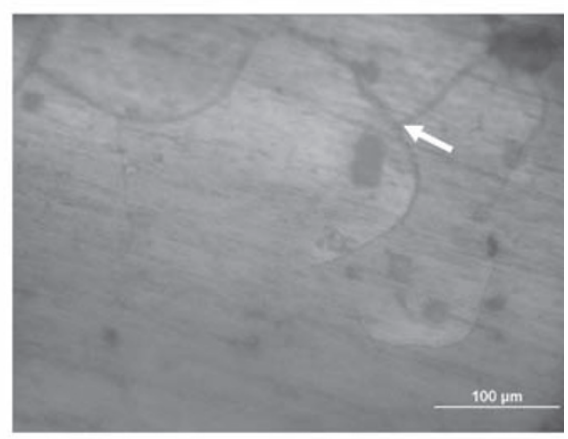

$\mathbf{f}$

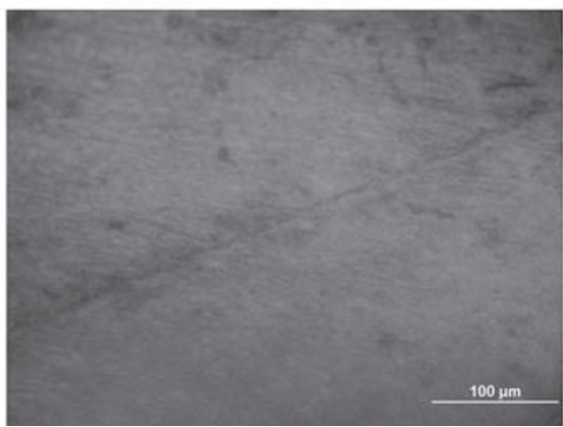

Figure 7 Micrographs of polystyrene, functionalized polystyrene and grafted polymer (PS, PSF1 and PSF1g, respectively) coated on metal surface at 0 $(\mathbf{a}, \mathbf{c}, \mathbf{e})$ and 10 days (b, d, f) while exposed to $3 \% \mathrm{NaCl}$ solution. Arrows indicate defects.

group), $3.14\left(-\mathrm{CH}<\right.$ grafted main chain of DIPF), $1.25\left(-\mathrm{CH}_{3}\right.$, isopropyl group) and other signals similar to Figure $3 \mathrm{a}$.

The final conversion was $66 \%$, and the degree of grafting was $320 \%$. This result is in accordance to the reactivity of this kind of monomer, which exhibited a much greater tendency to copolymerize rather than to homopolymerize ${ }^{24}$ because of the strong steric hindrance of the homopropagation step. The absence of the homopolymer during the grafting reaction was verified by SEC analysis. Figure $4 \mathrm{~b}$ presents the chromatogram of PSF1g using double detection (UV and IR). It can be seen that the chromatogram exhibited an unimodal distribution and two significant signals, indicating that both signals correspond to the same macromolecule. If the homopolymer (polydiisopropyl fumarate, PDIPF) had been formed, a bimodal chromatogram will be observed. In this case, a population assigned to the graft copolymer will display a signal in the UV and IR spectra, whereas the size distribution of PDIPF (homopolymer free) will be registered only by the IR detector. The evaluated weight-average molecular weight and polydispersity index of graft copolymer were $65400 \mathrm{~g} \mathrm{~mol}^{-1}$ and 2.4, respectively.
When the reaction was carried out under thermal conditions $\left(85^{\circ} \mathrm{C}\right.$ for $\left.20 \mathrm{~h}\right)$, a reduction in both conversion and degree of grafting (58 and 260\%, respectively) was observed. The SEC analysis of this polymer (Figure 6) show an increase of the average molecular weight and the polydispersity index, with $M_{\mathrm{w}}=77590 \mathrm{~g} \mathrm{~mol}^{-1}$ and $M_{\mathrm{w}} / M_{\mathrm{n}}=2.8$, with a tail toward higher molecular weights. This behavior could be due to a lower proportion of high-molecular weight homopolymer, but this aspect of the grafting reaction under thermal conditions is unclear and will be further investigated. These results highlight the greater efficiency of using microwave energy on the grafting reaction.

\section{Evaluation of the corrosion resistance}

The corrosion resistance properties of PS1, PSF1 and PSF1g on steel surfaces were evaluated for 10 days in $3 \%(\mathrm{w} / \mathrm{v}) \mathrm{NaCl}$ solutions, which simulate the sea conditions following the methodology by Ahmetli et al. ${ }^{25}$ Figure 7 shows the micrographs before and after the corrosion resistance analysis. The three polymers formed a transparent film on the metal, as seen in Figures $7 \mathrm{a}, \mathrm{c}$ and e. However, after 10 days of 
exposure to the conditions indicated above, significant differences were observed. The surface coating by PS presented great fissures and partly detached from the surface, shown in Figure 7b. The film of PSF showed only some small cracks, but much improved adhesion to the surface relative to the PS film, shown in Figure 7d. The PSF1g film showed a seamless surface and the best adhesion to the metal in the three types of polymers, shown in Figure $7 \mathrm{f}$. These results show that the derivatization reactions carried out using PS improves the anticorrosion properties of the films, making them suitable materials for protective coating.

\section{Conclusions}

A new methodology of Friedel-Crafts acylation with MA under mild conditions using $\mathrm{ZnO}$ and microwave energy as an energy source was successfully applied to functionalize polystyrene. No degradation was observed under the conditions used. The main advantages include the low cost, non-toxicity and reusability of the catalyst after simple washing with $\mathrm{Cl}_{2} \mathrm{CH}_{2}$, short reaction time and energy savings.

The functionalization introduced by MA as the acylating agent allows the subsequent preparation of graft copolymer by radical polymerization of vinyl monomers using microwave energy as an efficient energy source. This type of methodology could be successfully applied to other polymers that contain aromatic rings in its main chain.

Significant improvements were observed in the anticorrosive properties of these materials as a result of the chemical structure of the macromolecules.

\section{ACKNOWLEDGEMENTS}

Financial support from the Universidad Nacional de La Plata, Argentina, is acknowledged.

1 Kurbanova, R., Okudan, A., Mirzaoglu, R., Kurbanov, S., Karatas, I., Ersöz, M., Özcan, E., Ahmedova, G. \& Pamuk, V. Effects of the functional groups of polystyrene on its adhesion improvement and corrosion resistance. J. Adhes. Sci. Technol. 12, 947-955 (1998).

2 Higgins, J. S., Tambasco, M. \& Lipson, J. E. G. Polymer blends; stretching what we can learn through the combination of experiment and theory. Prog. Polym. Sci. 30, 832-843 (2005).

3 Dong, J. Y., Wang, Z. M., Hong, H. \& Chung, T. C. Synthesis of isotactic polypropylene containing a terminal $\mathrm{Cl}, \mathrm{OH}$, or $\mathrm{NH}_{2}$ group via metallocene-mediated polymerization/ chain transfer reaction. Macromolecules 35, 9352-9359 (2002).

4 Chen, J., Yang, G., Zhang, H. \& Chen, Z. A review: non-cross-linked polystyrene bound reagents, catalysts and syntheses. React. Funct. Polym. 66, 1434-1451 (2006).
5 Jang, B. B., Lee, K., Kwon, W. J. \& Suh, J. Binding of uranyl ion by 2,2'-dihydroxyazobenzene attached to a partially chloromethylated polystyrene. J. Polym. Sci. Part A Polym. Chem. 37, 3169-3177 (1999).

6 Fernyhough, C. M., Young, R. N., Ryan, A. J. \& Hutchings, L. R. Synthesis and characterisation of poly(sodium 4-styrenesulfonate) combs. Polymer 47, 3455-3463 (2006).

7 Kurbanova, R., Mirzaoglu, R., Akovali, G., Rzaev, Z. M. O., Karatas, I. \& Okudan, A. Side-chain functionalization of polystyrene with maleic anhydride in the presence of lewis acids. J. Appl. Polym. Sci. 59, 235-241 (1996).

$8 \mathrm{Li}, \mathrm{J}$. \& Li, H. M. Functionalization of syndiotactic polystyrene with succinic anhydride in the presence of aluminum chloride. Eur. Polym. J. 41, 823-829 (2005).

9 Zhang, G., Liu, L., Wang, H. \& Jiang, M. Preparation and association behavior of diblock copolymer ionomers based on poly(styrene-b-ethylene-co-propylene). Eur. Polym. J. 36, 61-68 (2000).

10 Kenyon, W. O. \& Waugh, G. P. Polyvinylacetophenone: preparation, reactions, and depolymerization. J. Polym. Sci. 32, 83-88 (1958).

11 Blanchette, J. A. \& Cotman, J. D. Synthesis of polyfunctional polymers. J. Org. Chem. 23, 1117-1122 (1958).

12 Wang, Q. L., Ma, Y., Ji, X., Yan, H. \& Qiu, Q. Regioselective acylation of anisole with carboxylic-acid over HZSM-5 catalyst. J. Chem. Soc. Chem. Commun. 22, 2307-2308 (1995).

13 Bond, G., Gardner, J. A., McCabe, R. W. \& Shorrock, D. J. Friedel-Crafts acylation reactions using heterogeneous catalysts stimulated by conventional and microwave heating. J. Mol. Catal. A Chem. 278, 1-5 (2007).

14 Thakuria, H., Borah, B. M. \& Das, G. Macroporous metal oxides as an efficient heterogeneous catalyst for various organic transformations-a comparative study. J. Mol. Catal. A Chem. 274, 1-10 (2007).

15 Bogdal, D., Penczek, P., Pielichowski, J. \& Prociak, A. Microwave assisted synthesis, crosslinking, and processing of polymeric materials. Adv. Polym. Sci. 163, 194-263 (2003).

16 Sinnwell, S. \& Ritter, H. Recent advances in microwave-assisted polymer synthesis. Aust. J. Chem. 60, 729-743 (2007).

17 Cortizo, M. S. Polymerization of diisopropyl fumarate under microwave irradiation. J. Appl. Polym. Sci. 103, 3785-3791 (2007).

18 Oberti, T. G., Schiavoni, M. M. \& Cortizo, M. S. Structure and properties of poly(benzyl acrylate) synthesized under microwave energy. Rad. Phys. Chem. 77, 597-604 (2008).

19 Sarvari, M. H. \& Sharghi, H. Zinc oxide ( $\mathrm{ZnO}$ ) as a new, highly efficient, and reusable catalyst for acylation of alcohols, phenols and amines under solvent free conditions. Tetrahedron 61, 10903-10907 (2005).

20 Cortizo, M. S., Andreetta, H. A. \& Figini, R. V. Molecular characterization of poly(diisopropylfumarate) by the absolute calibration method in molecular exclusion chromatography (GPC). J. High Reslout. Chromatogr. Chromatogr. Comm. 12, 372-374 (1989).

21 Cianga, I., Mercore, V. M., Grigoras, M. \& Yagci, Y. Synthesis and characteristics of polymacromonomers composed of alternating binaphthalene-phenylene main chain and polystyrene side chains. Polymer 48, 6501-6509 (2007).

22 Zheng, Y., Li, Y., Pan, L. \& Li, Y. Synthesis of novel functional polyolefin containing carboxylic acid via Friedel-Crafts acylation reaction. Polymer 48, 2496-2502 (2007).

23 Hird, B. \& Eisenberg, A. p-carboxylation of linear high molecular-mass polystyrene. J. Polym. Sci. Part A Polym. Chem. 31, 1377-1381 (1993).

24 Otsu, T., Matsumoto, A., Shiraishi, K., Amaya, N. \& Koinuma, Y. Effect of the substituents on radical copolymerization of dialkyl fumarates with some vinyl monomers. J. Polym. Sci. Part A Polym. Chem. 30, 1559-1565 (1992).

25 Ahmetli, G., Sen, N., Pehlivan, E. \& Durak, S. Adhesive and anticorrosive polymeric coatings obtained from modified industrial waste oligostyrenes. Prog. Org. Coat. 55, 262-267 (2006).

Supplementary Information accompanies the paper on Polymer Journal website (http://www.nature.com/pj) 